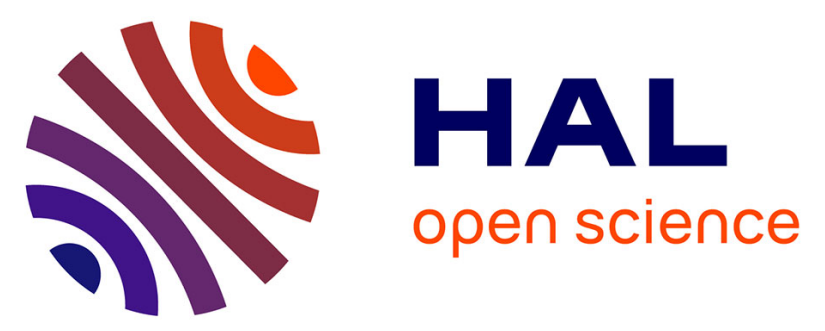

\title{
JAK2V617F mutation persists in blasts and mature cells of transformed- JAK2V617F-positive-myeloproliferative neoplasia: a European Leukemia Net (ENL) study.
}

Ciro Roberto Rinaldi, Paola Rinaldi, Marica Gemei, Francesco Grimaldi, Giorgia Battipaglia, Luigi del Vecchio, Bruno Martino, Giorgina Specchia, Anna Candoni, Luigi Gugliotta, et al.

\section{To cite this version:}

Ciro Roberto Rinaldi, Paola Rinaldi, Marica Gemei, Francesco Grimaldi, Giorgia Battipaglia, et al. JAK2V617F mutation persists in blasts and mature cells of transformed- JAK2V617F-positivemyeloproliferative neoplasia: a European Leukemia Net (ENL) study.. American Journal of Hematology, 2010, 10.1002/ajh.21687 . hal-00552311

\section{HAL Id: hal-00552311 \\ https://hal.science/hal-00552311}

Submitted on 6 Jan 2011

HAL is a multi-disciplinary open access archive for the deposit and dissemination of scientific research documents, whether they are published or not. The documents may come from teaching and research institutions in France or abroad, or from public or private research centers.
L'archive ouverte pluridisciplinaire HAL, est destinée au dépôt et à la diffusion de documents scientifiques de niveau recherche, publiés ou non, émanant des établissements d'enseignement et de recherche français ou étrangers, des laboratoires publics ou privés. 


\section{American Journal of Hematology}

WILEY

\section{JAK2V617F mutation persists in blasts and mature cells of transformed- JAK2V617F-positive-myeloproliferative neoplasia: a European Leukemia Net (ENL) study.}

\begin{tabular}{|c|c|}
\hline Journal: & American Journal of Hematology \\
\hline Manuscript ID: & AJH-10-0089 \\
\hline Wiley - Manuscript type: & Letters \\
\hline $\begin{array}{r}\text { Date Submitted by the } \\
\text { Author: }\end{array}$ & $10-$ Feb-2010 \\
\hline Complete List of Authors: & $\begin{array}{l}\text { rinaldi, ciro; Federico II University, Hematology and CEINGE } \\
\text { Rinaldi, Paola; Federico II University, Hematology } \\
\text { Gemei, Marica; Federico II University, Hematology } \\
\text { Grimaldi, Francesco; Federico II University, Hematology } \\
\text { Battipaglia, Giorgia; Federico II University, Hematology } \\
\text { Del Vecchio, Luigi; Federico II University, Hematology } \\
\text { Martino, Bruno; Ospedale di Reggio Calabria, Hematology } \\
\text { Specchia, Giorgina; University of Bari, Hematology } \\
\text { Candoni, Anna; University of Udine, Hematology } \\
\text { Gugliotta, Luigi; Ospedale di Reggio Emilia, Hematology } \\
\text { Vannucchi, Alessandro; University of Firenze, Hematology } \\
\text { Barbui, Tiziano; Ospedali Riuniti, Hematology } \\
\text { Pane, Fabrizio; Federico II University, Hematology }\end{array}$ \\
\hline Keywords: & $\begin{array}{l}\text { JAK2V617F mutation, secondary leukemia, myeloproliferative } \\
\text { neoplasia }\end{array}$ \\
\hline
\end{tabular}

\section{S scholaroNE" \\ Manuscript Central}




\title{
JAK2 V617F mutation persists in blasts and mature cells of transformed- JAK2V617F-positive-myeloproliferative neoplasia: a European Leukemia Net (ENL) study.
}

\begin{abstract}
Ciro R. Rinaldi ${ }^{1}$, Paola Rinaldi ${ }^{1}$, Marica Gemei $^{1}$, Francesco Grimaldi ${ }^{1}$, Giorgia Battipaglia $^{1}$, Luigi Del Vecchio ${ }^{1}$, Bruno Martino $^{2}$, Giorgina Specchia ${ }^{3}$, Anna Candoni ${ }^{4}$, Luigi Gugliotta $^{5}$, Alessandro M. Vannucchi ${ }^{6}$, Tiziano Barbui ${ }^{7}$ and Fabrizio Pane ${ }^{1}$

${ }^{1}$ Hematology Division, Department of Biochemistry and Medical Biotechnology, University Federico II, Naples, Italy; ${ }^{2}$ Hematology, Reggio Calabria Hospital, Reggio Calabria, Italy; ${ }^{3}$ Division of Hematology, University of Bari, Italy; ${ }^{4}$ Division of Hematology, Bone Marrow Transplantation Unit, University of Udine, Udine, Italy; ${ }^{5}$ Department of Hematology, Arcispedale Santa Maria Nuova, Reggio Emilia, Italy; ${ }^{6}$ Department of Hematology, University of Florence, Florence, Italy; ${ }^{7}$ Hematology, Ospedali Riuniti di Bergamo, Bergamo, Italy.
\end{abstract}

Running Title: JAK2V617F mutation in secondary acute myeloid leukemia

Keywords: JAK2V617F mutation, secondary leukemia, myeloproliferative neoplasia

Word count: Abstract 149; Text 1440; Tables 2

Correspondence: Dr. Ciro Roberto RINALDI

Hematology Division - Federico II University

Via Pansini 5, 80131 Naples - Italy

Email: rinaldic@dbbm.unina.it

This article is dedicated to the memory of Prof. Bruno ROTOLI 
To the Editor.

Transformation to acute myeloid leukemia (AML) is a known complication of myeloproliferative neoplasia (MPN). Recent studies reported the high incidence (53\%) of JAK2 negative blasts from transformed JAK2V617F-MPN. We collected, by cell sorting, blast cells and mature cells (GRA) from total bone marrow (BM) of 34 patients newly diagnosed of secondary AML. At MPN diagnosis (PMF n = 18; PV n = 9; ET n = 7), JAK2 was mutated in 22 of 34 patients. Twenty of 22 JAK2V617F-MPN (91\%) maintained the mutation in blasts and GRA after leukemic switch, while in 2 of 22 patients the selected compartments lost the mutation. Surprisingly we also found the first case of JAK2V617FAML from a wild type (WT)-MPN. In contrast to the previous study, we conclude that JAK2V617F-MPN yields rarely (9\%) a JAK2WT-AML and any JAK2-status modification/persistence involves always the entire BM during leukemic transformation.

Myeloproliferative neoplasia (MPN) are a heterogeneous group of diseases characterized by the hyperproliferation of myeloid precursors in the bone marrow (BM) and/or in extra-medullary sites leading to elevated number of non-lymphoid cells and/or platelets in the peripheral blood[1]. The discovery of an acquired somatic mutation in the $J A K 2$ gene resulting in a valine-tophenylalanine substitution at position $617(J A K 2 \mathrm{~V} 617 \mathrm{~F})$ has substantially modified the molecular knowledge's behind these syndromes[2-5]. By a sensitive allele-specific polymerase chain reaction (ASO-PCR) assay[6], it has been well established that around 97\% of patients affected by polycythemia vera (PV), 60-70\% of patients with essential thrombocythemia (ET), and $60 \%$ of primary myelofibrosis (PMF) are positive for the $J A K 2 \mathrm{~V} 617 \mathrm{~F}$ mutation. Transformation to acute myeloid leukemia (AML) is a known complication of MPN but the role of JAK2V617F mutation is still undefined [7-14]. In 2006, Campbell analyzed 4 patients with secondary AML 
describing a possible model for the development of a JAK2WT-AML in a patient with JAK2V617F-MPN[10] and more recently, Theocharides et al. reported that in up to 53\% of the patients who developed secondary AML from a JAK2-mutated MPN the mutation was no longer detectable; however the results, in a smaller cohort of patients $(n=27)$, obtained with DNA extracted from cells scraped or laser-capture-microdissected from bone marrow (BM) or peripheral blood (PB) smears, were confirmed in fresh samples only in some cases with nouniform cell sources (PB granulocytes, BM-blasts, PB-blasts) [15]. In this study, we collected, by cell sorting, blast cells and mature myeloid cells (granulocytes, GRA) from whole BM aspirates of 34 newly diagnosed patients with AML secondary to MPN (18 derived from PMF; 9 from PV and 7 from ET) and analyzed the $J A K 2$ status before and after leukemic transformation in selected cell compartments. In contrast to the previous studies, we conclude that a JAK2V617Fpositive-MPN yields rarely (9\%) a JAK2V617F-negative-AML. At the time of MPN diagnosis, JAK2V617F was detectable in 22 of 34 patients (65\%) (10 of 18 PMF; 9 of 9 PV and 3 of 7 ET) (Table 1). Information on treatment during the chronic phase was available in 25 of 34 patients. Twenty patients had received cytoreductive treatment with hydroxyurea $(\mathrm{HuOH}), 2$ patients with $\mathrm{HuOH}$ associated to busulphan, 1 patient with IFN-alpha, 1 patient with busulphan plus anagrelide, 1 patient $\mathrm{HuOH}$ plus clorambucil (Table 1). Four PV-untreated-patients had a spontaneous transformation to AML. No cytogenetic abnormalities were detected during the chronic phase of the disease in 30/34 evaluated patients. No MPL or JAK2-exone 12 mutations were present at this stage (data not shown). Median time to AML progression (TTP) was 5.09 years $(y r s)$ (range $0.38-27.81)$. A significant difference $(p=0.02)$ in TTP was found grouping patients according to JAK2 status during the MPN phase [JAK2WT-MPN n=12, TTP median 15.10 yrs (0.38-16.32); JAK2 mutated-MPN n=22, TTP median 4.07 yrs (0.67-27.81)]. Cytogenetic data were available in 23 of 34 patients during AML transformation: 8 patients 
showed additional abnormalities involving chromosomes 1, 5, 7, 8, 9, 12, 14, 17 and 20 (Table 2) while normal karyotype was confirmed in 14 patients. No other AML-associated mutations (FLT3, NPM, CEBPA, RUNX1) were detectable at this stage. To evaluate the modification in the $J A K 2$ status before and during leukemic transformation we performed ASO-PCR and (QRT)PCR assay on total BM of the MPN phase and sorted cell populations from AML phase. No JAK2V617F mutation was detectable in sorted CD3+ cells (Table 2). In our cohort of patients we found that $J A K 2 \mathrm{~V} 617 \mathrm{~F}$ mutation was still present at the blast transformation in both compartments: CD34+ cells (blasts) and CD15+ cells (GRA) in 20 of 22 JAK2 mutated MPN (91\%) (Table 2). Two of 22 patients (9\%) developed JAK2V617F negative AML starting from a mutated PV with a mean TTP of 5.14 yrs. Interestingly, the WT status was confirmed in blast cells but also in GRA (Table 2). Surprisingly we found a case of JAK2V617F mutated AML transforming from a WT-PMF. The negativity for the mutation at MPN stage was confirmed in two different laboratories using 3 different PCR assays. Also in this case the JAK2V617F positivity in the AML phase occurred in both GRA and blast compartments with an allele burden ratio of $42 \%$ and $41 \%$ respectively. The remaining 11 JAK2WT-MPN maintained the same JAK2 status during blast crisis. To determine whether leukemic transformation could modify the JAK2mutational burden we performed (QRT)-PCR assay. For patients with persisting JAK2-mutated disease $(n=20)$, no differences $(p=0.3)$ in the allele burden were found comparing MNCs from chronic phase (allelic ratio, median $\pm S D: 57 \% \pm 1.75$ ) with MNCs of leukemic transformations (allelic ratio, median $\pm S D: 56 \% \pm 1.75)$. No differences in the allele burden $(\mathrm{p}=0.4)$ were also found comparing GRA (allelic ratio, median $\pm S D: 56 \% \pm 2.5$ ) with blasts (allelic ratio, median $\pm S D: 56 \% \pm 1.5)$ in AML phase. These results contrast with previous studies in which the $J A K 2 \mathrm{~V} 617 \mathrm{~F}$ mutation levels decreased during leukemic evolution [19, 20] (data summarized in Table 2). At the time of the study, 6 of 34 patients were eligible for intensive chemotherapy: 4 of 
6 patients achieved complete remission after induction treatment: 3 JAK2mutated-AML from JAK2mutated-MPN (2 ET and $1 \mathrm{PV}$ ); 1 JAK2WT-AML from JAK2mutated-PMF; 2 JAK2WTAML from JAK2WT-MPNs obtained partial remission. Twenty-three patients underwent palliative treatment while five deceased before starting cytotoxic treatment. One patient, $J A K 2$ mutated-AML from JAK2mutated-ET (patient 9), underwent allogeneic stem cell transplantation from sibling donor and achieved a stable remission. Remarkably, the JAK2V617F mutation was no longer detectable by quantitative PCR.

In conclusion, these results contrast with the previous study in which the $J A K 2$ mutation was lost in $53 \%$ of blasts during leukemia transformation. In our work, the loss of JAK2V617F mutation during AML progression is a rare event $(9 \%)$. One possible explanation may relate to the different approach used by Theocharides et al [15] who first isolated DNA from cells collected using laser-dissection or brushing from stored BM or PB smears and then confirmed the results in half of patients in fresh isolated cells. It can be possible, in theory, that a poor qualityDNA could not be efficiently amplified by using mutation-specific, single-base mismatched primers while amplification of wild-type sequence could have been favored. Furthermore in several cases laser dissection could have collected a non-representative part of the leukemic clone considering the non-homogenous distribution in BM or PB smears implying an underestimation of the JAK2 mutated blasts which could have been diluted in a majority of WT cells and not detected by conventional PCR. For those cases in which the results were confirmed in fresh cells the source of the cells was often represented by PB-CD34+ cells collected using non comparable techniques (FACS or MACS), sometimes mixing $\mathrm{PB}$ and $\mathrm{BM}$ blasts but no additional information about number and quality of the cells were provided. When the type of MPN studied is taken into account one aspect seems of interest: no mutated-PMF patients $(\mathrm{n}=10)$, who 
represent the majority of our mutated cases, lost the $J A K 2 \mathrm{~V} 617 \mathrm{~F}$ mutation during leukemia. PMF patients are much more likely to transform than ET and PV, which represent a more benign diseases also more frequently $J A K 2$-mutated, but surprisingly in this view the only 2 patients changing the JAK2 status after transformation were PV. This finding is in accordance with recently published data [17] that demonstrated how JAK2-mutant AML, but not JAK2-WT AML, usually arises from a preceding myelofibrotic phase. This allow to think that probably the mechanisms of leukemic transformation are different in PMF comparing with ET and PV and that the JAK2V617F status of AML secondary to PMF may play a different role respect to the JAK2V617F status of AML secondary to ET and PV. One PMF patient developed a secondary mutated-AML from a WT disease. Both ASO-PCR and real time assays excluded the presence of JAK2V617 mutation during the chronic phase of the MPN in both BM cells and in peripheral GRA, reinforcing strongly the hypothesis that the mutation could have been acquired during leukemia switch as it has been reported to occur in 3-4\% of de novo AML [18]. However, the possibility that a very low burden of JAK2 mutated allele during the chronic phase may have gone undetected cannot be ruled out. One additional finding resulting from this study is that presence of the $J A K 2 \mathrm{~V} 617 \mathrm{~F}$ mutation during the MPN phase seems to be associated with an acceleration to leukemic transformation compared to WT disease, although the incidence of AML in the two groups was comparable. Additional studies in larger patient series and multivariate analysis are needed before a prognostic role of $J A K 2 \mathrm{~V} 617 \mathrm{~F}$ mutation regarding time to leukemia transformation can be definitely assessed. However the persistence of the JAK2V617F mutation in the actually largest patient series provides a new intriguing scenery that

opens again the discussion. 


\section{PATIENTS AND METHODS}

Patients. We performed the molecular analysis on $34 \mathrm{BM}$ aspirates obtained by patients newly diagnosed with secondary AML (18 derived from PMF; 9 from PV and 7 from ET). Informed consent for the study from all patients was obtained in accordance with the Declaration of Helsinki. The study was conducted according to the guidelines of Italian local ethics committee. The diagnosis of MPN was made according to WHO[16]. MPN-secondary-AML was diagnosed according to WHO criteria using $20 \%$ blasts in BM as cut off. Patient's characteristics are summarized in Table 1.

Cell Sorting and DNA preparation. Mononucleated cells (MNCs) were isolated by Ficoll centrifugation. After erythrocyte lyses, 10 million BM (or PB) cells were labelled by incubation with the antibodies CD15-APC, CD3-FITC, CD45-PerCP, CD34-Pe (BD Biosciences, San Jose, CA), analyzed and sorted with a fluorescence-activated cell sorter FACSAria (BD Biosciences) using FACS Diva software (BD Biosciences), according to manufacturer's recommendations. Leukemic blasts were collected using side scatter according to CD45 and CD34 expression. Mature cells (GRA) and T-cell were sorted by FACS using CD15 and CD3 antibodies, respectively. Leukemic blasts and GRA were $\geq 98 \%$ pure by morphological criteria and FACS reanalysis. Genomic DNA was isolated using QIAmp DNA Blood Kit (Qiagen).

ASO-PCR and QRT-PCR. The JAK2V617F mutation status was determined by ASO-PCR as previously described [2] with a sensitivity of $1 \%$. Measurement of $J A K 2 \mathrm{~V} 617 \mathrm{~F}$ allele burden was performed by a quantitative real time (QRT)-PCR assay, using 80 ng DNA. PCR amplification and detection were performed on an ABI Prism 7300 analyzer (Applied Biosystem) using the following cycling conditions: 10 minutes at $95^{\circ} \mathrm{C}$ followed by 50 cycles of $15 \mathrm{~s}$ at $95^{\circ} \mathrm{C}$ and $60 \mathrm{~s}$ 
at $60^{\circ} \mathrm{C}$. QRT-PCR assay was performed with specific forward primers [JAK2-wild type (WT): 5'-GCGCGGTTTAAATTATGGAGTATGTG-3'; JAK2V617F: 5'GCGCGGTTTTAAATTATGGAGTATGTT-3'), common reverse primers (5'GCGGTGATCCTGAAACTGAATTTTC-3'). FAM-labeled MGB probe sequence was: 5'TGGAGACGAGAGTAAGTAAAACTACAGGCT-3'. All samples were analyzed in triplicate and the amount of $J A K 2 \mathrm{~V} 617 \mathrm{~F}$ allele was calculated by comparison with cloned JAK2 plasmids. The mean of triplicate $\triangle \mathrm{CT}$ determinations $\left(\mathrm{C}_{\mathrm{T}}^{\text {JAK2V617F}}-\mathrm{C}_{\mathrm{T}}{ }^{J A K 2 \mathrm{WT}}\right)$ was used to calculate the percentage of mutated allele. Positive and negative controls were included in each assay; interand intra-assay variation was $3 \%$ and $5 \%$, respectively[5]. To compare continuous variables among the groups we used the Mann-Whitney $U$ test and Student's t-Test.

\section{Acknowledgments}

This work was conducted on behalf of the European Leukemia Net (ENL).

\section{REFERENCES}

1. Vardiman JW, Harris NL, Brunning RD. The World Health Organization (WHO) classification of the myeloid neoplasms. Blood. 2002 Oct 1;100(7):2292-302.

2. Baxter EJ, Scott LM, Campbell PJ, et al. Acquired mutation of the tyrosine kinase JAK2 in human myeloproliferative disorders. Lancet. 2005 Mar 19-25;365(9464):1054-61.

3. Kralovics R, Passamonti F, Buser AS, et al. A gain-of-function mutation of JAK2 in myeloproliferative disorders. N Engl J Med. 2005 Apr 28;352(17):1779-90. 
4. Levine RL, Wadleigh M, Cools J, et al. Activating mutation in the tyrosine kinase JAK2 in polycythemia vera, essential thrombocythemia, and myeloid metaplasia with myelofibrosis. Cancer Cell. 2005 Apr;7(4):387-97.

5. Lippert E, Boissinot M, Kralovics R, et al. The JAK2-V617F mutation is frequently present at diagnosis in patients with essential thrombocythemia and polycythemia vera. Blood. 2006 Sep 15;108(6):1865-7.

6. Veneri D, Capuzzo E, de Matteis G, et al. Comparison of JAK2V617F mutation assessment employing different molecular diagnostic techniques. Blood Transfus. 2009 Jul;7(3):204-9.

7. Schnittger S, Bacher U, Kern W, et al. JAK2V617F as progression marker in CMPD and as cooperative mutation in AML with trisomy 8 and $\mathrm{t}(8 ; 21)$ : a comparative study on $1103 \mathrm{CMPD}$ and 269 AML cases. Leukemia. 2007 Aug;21(8):1843-5.

8. Rossi D, Deambrogi C, Capello D, et al. JAK2 V617F mutation in leukaemic transformation of philadelphia-negative chronic myeloproliferative disorders. $\mathrm{Br} \mathrm{J}$ Haematol. 2006 Oct;135(2):267-8.

9. Panovska-Stavridis I, Cevreska L, Ivanovski M, et al. JAK2V617F mutations in myeloid malignancies: single center experience. Prilozi. 2009 Dec;29(2):257-68.

10. Campbell PJ, Baxter EJ, Beer PA, et al. Mutation of JAK2 in the myeloproliferative disorders: timing, clonality studies, cytogenetic associations, and role in leukemic transformation. Blood. 2006 Nov 15;108(10):3548-55.

11. Frohling S, Lipka DB, Kayser S, et al. Rare occurrence of the JAK2 V617F mutation in AML subtypes M5, M6, and M7. Blood. 2006 Feb 1;107(3):1242-3.

12. Hsiao HH, Yang WC, Liu YC, et al. Disappearance of JAK2 V617F mutation in a rapid leukemic transformed essential thrombocythemia patient. Leuk Res. 2008 Aug;32(8):1323-4. 
13. Iwanaga E, Nanri T, Matsuno N, et al. A JAK2-V617F activating mutation in addition to KIT and FLT3 mutations is associated with clinical outcome in patients with $\mathrm{t}(8 ; 21)(\mathrm{q} 22 ; \mathrm{q} 22)$ acute myeloid leukemia. Haematologica. 2009 Mar;94(3):433-5.

14. Lee JW, Kim YG, Soung YH, et al. The JAK2 V617F mutation in de novo acute myelogenous leukemias. Oncogene. 2006 Mar 2;25(9):1434-6.

15. Theocharides A, Boissinot M, Girodon F, et al. Leukemic blasts in transformed JAK2V617F-positive myeloproliferative disorders are frequently negative for the JAK2-V617F mutation. Blood. 2007 Jul 1;110(1):375-9.

16. Tefferi A, Thiele J, Vardiman JW. The 2008 World Health Organization classification system for myeloproliferative neoplasms: order out of chaos. Cancer. 2009 Sep 1;115(17):38427.

17. Beer PA, Delhommeau F, Lecouedic JP, et al. Two routes to leukemic transformation following a JAK2 mutation-positive myeloproliferative neoplasm. Blood. 2009 Dec 11.

18. Lee JW, Kim YG, Soung YH, et al. The JAK2 V617F mutation in de novo acute myelogenous leukemias. Oncogene. 2006 Mar 2;25(9):1434-6.

19. Zhang SJ, Li JY, Zhang JF, et al. The decrease of JAK2 V617F allele burden in leukemia transformation of an elderly patient with myelofibrosis. Leuk Res. 2009 Aug;33(8):e116-8.

20. Hussein K, Bock O, Theophile K, et al. JAK2(V617F) allele burden discriminates essential thrombocythemia from a subset of prefibrotic-stage primary myelofibrosis. Exp Hematol. 2009 Jul 17. 


\section{Authorship and disclose}

Contribution: C.R.R. performed research, analyzed data, and wrote the paper; P.R., F.G.and G.B. performed research and analyzed data; B.M., G.S., A.C., L.G. provided patients; M.G., L.D.V. performed the FACS sorting; A.V. performed real time PCR; T.B. and F.P. designed the research and wrote the paper. The authors have no conflict of interest to disclose. 
Table 1. Summary of patient characteristics: MPN diagnosis and previous treatment

\begin{tabular}{|c|c|c|c|c|c|c|}
\hline patient & gender & age & diagnosis & $J A K 2$ & cytoreductive treatment & $\operatorname{TTP}(y r s)$ \\
\hline 1 & $\bar{M}$ & 72 & PMF & $\overline{\text { WT }}$ & $\mathrm{HuOH}$ & 16.42 \\
\hline 2 & M & 67 & ET & WT & $\mathrm{BU}+\mathrm{HuOH}$ & 15.43 \\
\hline 3 & $\mathrm{~F}$ & 67 & PMF & WT & $\mathrm{BU}+\mathrm{HuOH}$ & 20.3 \\
\hline 4 & M & 35 & PMF & WT & - & 6.01 \\
\hline 5 & $\mathrm{~F}$ & 32 & PMF & WT & $\mathrm{HuOH}$ & 5.09 \\
\hline 6 & M & 68 & PMF & MUT & $\mathrm{HuOH}$ & 2.01 \\
\hline 7 & $\mathrm{~F}$ & 70 & ET & MUT & $\mathrm{HuOH}$ & 4.71 \\
\hline 8 & $\mathrm{~F}$ & 88 & PMF & MUT & $\mathrm{HuOH}$ & 27.81 \\
\hline 9 & M & 66 & PV & MUT & $\mathrm{HuOH}$ & 4.99 \\
\hline 10 & M & 45 & ET & MUT & IFN & 2.64 \\
\hline 11 & $\mathrm{~F}$ & 57 & PV & MUT & $\mathrm{HuOH}$ & 4.71 \\
\hline 12 & $\mathrm{~F}$ & 59 & PMF & MUT & - & 1.00 \\
\hline 13 & M & 55 & PV & MUT & None & 5.98 \\
\hline 14 & $\mathrm{~F}$ & 74 & PV & MUT & None & 7.51 \\
\hline 15 & $\mathrm{~F}$ & 77 & PMF & WT & Clor $+\mathrm{HuOH}$ & 16.32 \\
\hline 16 & M & 74 & PMF & WT & $\mathrm{HuOH}$ & 4.13 \\
\hline 17 & $\mathrm{~F}$ & 81 & ET & MUT & - & 1.00 \\
\hline 18 & M & 58 & PMF & WT & $\mathrm{HuOH}$ & 0.38 \\
\hline 19 & $\mathrm{~F}$ & 68 & PMF & MUT & $\mathrm{HuOH}$ & 13.16 \\
\hline 20 & $\mathrm{~F}$ & 65 & PV & MUT & None & 1.02 \\
\hline 21 & $\mathrm{~F}$ & 60 & PMF & MUT & $\mathrm{HuOH}$ & 5.96 \\
\hline 22 & M & 49 & PMF & MUT & $\mathrm{HuOH}$ & 1.01 \\
\hline 23 & $\mathrm{~F}$ & 71 & PMF & MUT & $\mathrm{HuOH}$ & 4.07 \\
\hline 24 & $\mathrm{~F}$ & 66 & ET & WT & $\mathrm{HuOH}$ & 15.11 \\
\hline 25 & $\mathrm{~F}$ & 70 & PMF & MUT & - & 11.09 \\
\hline 26 & $\mathrm{~F}$ & 77 & ET & WT & $\mathrm{HuOH}$ & 9.03 \\
\hline 27 & $\mathrm{~F}$ & 71 & PV & MUT & $\mathrm{HuOH}$ & 10.16 \\
\hline 28 & $\mathrm{~F}$ & 75 & PV & MUT & $\mathrm{HuOH}$ & 1.08 \\
\hline 29 & M & 81 & PMF & MUT & $\mathrm{HuOH}$ & 2.29 \\
\hline 30 & M & 83 & PV & MUT & None & 5.30 \\
\hline 31 & $\mathrm{~F}$ & 76 & PV & MUT & $\mathrm{HuOH}$ & 11.32 \\
\hline 32 & M & 65 & PMF & MUT & $\mathrm{HuOH}$ & 0.67 \\
\hline 33 & M & 64 & PMF & WT & - & 8.13 \\
\hline 34 & $\mathrm{~F}$ & 67 & ET & WT & BU+ANA & 1.09 \\
\hline
\end{tabular}

M, male; F, female; PMF, primary myelofibrosis, ET, essential thrombocytemia; PV, polycythemia vera; $\mathrm{HuOH}$, hydroxiurea; $\mathrm{BU}$, busulphan; IFN, interferon; ANA, anagrelide; Clor, clorambucil; (-), treatment information's not available; TTP, time to progression; yrs, years 
Page 13 of 13 able 2. Summary of patient characteristics: molecular analysis iAmerican socondaral of Hamatology

\begin{tabular}{|c|c|c|c|c|c|c|c|c|c|}
\hline \multirow[b]{2}{*}{ patient } & \multicolumn{2}{|r|}{ MPN } & \multicolumn{7}{|c|}{ AML } \\
\hline & diagnosis & $\begin{array}{c}J A K 2 \\
\operatorname{MNCs}(A B \% \pm S D)\end{array}$ & source & $\% B L A S T$ & cytogenetic & $\begin{array}{c}J A K 2 \\
\operatorname{MNCs}(A B \% \pm S D)\end{array}$ & $\begin{array}{c}J A K 2 \\
\text { BLAST }(A B \% \pm S D)\end{array}$ & $\begin{array}{c}J A K 2 \\
\text { CD15+ Cells }(A B \% \pm S D)\end{array}$ & $\begin{array}{c}J A K 2 \\
\text { CD3+ Cells }\end{array}$ \\
\hline 1 & PMF & WT & $\overline{B M}$ & 80 & ND & $\overline{\text { WT }}$ & WT & WT & WT \\
\hline 2 & ET & WT & BM & 90 & COMPLEX & WT & WT & WT & WT \\
\hline 3 & PMF & WT & BM & 95 & DEL(5)+TRI(8)+TRI(1) & WT & WT & WT & WT \\
\hline 4 & PMF & WT & BM & 70 & $\operatorname{DEL}(7)+\operatorname{DEL}(9)+\operatorname{TRI}(14)$ & WT & WT & WT & WT \\
\hline 5 & PMF & WT & BM & 70 & $\mathrm{ND}$ & MUT $(42 \pm 2.85)$ & MUT $(41 \pm 1.75)$ & MUT (42 \pm 2$)$ & WT \\
\hline 6 & PMF & MUT $(58 \pm 1.75)$ & BM & 76 & $46, \mathrm{XY}$ & MUT (57 \pm 2.5$)$ & MUT $(58 \pm 2)$ & MUT $(57 \pm 2.25)$ & WT \\
\hline 7 & ET & MUT $(55 \pm 1.51)$ & BM & 40 & $46, \mathrm{XX}$ & MUT (54 \pm 1.5$)$ & MUT (55 \pm 2$)$ & MUT $(55 \pm 1.25)$ & WT \\
\hline 8 & PMF & MUT $(57 \pm 2.00)$ & BM & 83 & $46, \mathrm{XX}$ & MUT $(56 \pm 1.75)$ & MUT $(58 \pm 2.5)$ & MUT $(57 \pm 0.75)$ & WT \\
\hline 9 & $\mathrm{PV}$ & MUT $(62 \pm 2.13)$ & BM & 71 & $46, X Y$ & WT & WT & WT & WT \\
\hline 10 & ET & MUT $(32 \pm 0.95)$ & BM & 72 & $46, X Y$ & MUT $(30 \pm 2.05)$ & MUT (32 \pm 1.5$)$ & MUT (35 \pm 1.5$)$ & WT \\
\hline 11 & $\mathrm{PV}$ & MUT $(60 \pm 1)$ & BM & 40 & $46, \mathrm{XX}$ & MUT $(61 \pm 1.05)$ & MUT $(60 \pm 1)$ & MUT (59 \pm 1.5$)$ & WT \\
\hline 12 & PMF & MUT (56 \pm 1.2$)$ & $\mathrm{PB}$ & 59 & MONO(7), TRI(1) & MUT $(57 \pm 2)$ & MUT (56 \pm 2.5$)$ & MUT (56 \pm 2$)$ & WT \\
\hline 13 & PV & MUT $(59 \pm 2)$ & BM & 78 & $\mathrm{ND}$ & MUT $(55 \pm 1.75)$ & MUT $(60 \pm 1.5)$ & MUT $(59 \pm 1.75)$ & WT \\
\hline 14 & PV & MUT (81 \pm 3$)$ & BM & 55 & ND & MUT (76 \pm 2$)$ & MUT $(71 \pm 2.75)$ & MUT (69 \pm 2.2$)$ & WT \\
\hline 15 & PMF & WT & BM & 90 & ND & WT & WT & WT & WT \\
\hline 16 & PMF & WT & BM & 62 & ND & WT & WT & WT & WT \\
\hline 17 & ET & MUT $(26 \pm 1.95)$ & BM & 27 & ND & MUT (21 \pm 3.5$)$ & MUT $(30 \pm 2.76)$ & MUT $(23 \pm 2.7)$ & WT \\
\hline 18 & PMF & WT & BM & 69 & $46, \mathrm{XY}$ & WT & WT & WT & WT \\
\hline 19 & PMF & MUT $(57 \pm 1.75)$ & BM & 60 & COMPLEX & MUT (57 \pm 2.5$)$ & MUT $(58 \pm 2)$ & MUT $(58 \pm 2)$ & WT \\
\hline 20 & $\mathrm{PV}$ & MUT $(56 \pm 1.15)$ & BM & 37 & 46, XX & MUT (55 \pm 2.5$)$ & MUT $(56 \pm 2)$ & MUT (55 \pm 2$)$ & WT \\
\hline 21 & PMF & MUT (57 \pm 1.6$)$ & BM & 85 & DEL(20) & MUT $(55 \pm 1.67)$ & MUT $(55 \pm 1.25)$ & MUT (56 \pm 1.5$)$ & WT \\
\hline 22 & PMF & MUT $(56 \pm 2.02)$ & BM & 60 & ND & MUT (56 \pm 0.75$)$ & MUT (55 51$)$ & MUT (56 \pm 1.2$)$ & WT \\
\hline 23 & PMF & MUT $(58 \pm 3.05)$ & BM & 100 & $46, X X$ & MUT (58 \pm 0.5$)$ & MUT $(57 \pm 1)$ & MUT $(57 \pm 1.97)$ & WT \\
\hline 24 & ET & WT & BM & 100 & $46, X X$ & WT & WT & WT & WT \\
\hline 25 & PMF & MUT (52 \pm 3.5$)$ & $\mathrm{PB}$ & 47 & 46, XX & MUT (50 \pm 1.5$)$ & MUT $(50 \pm 2)$ & MUT (51 \pm 2.3$)$ & WT \\
\hline 26 & ET & WT & BM & 24 & TRI(8) & WT & WT & WT & WT \\
\hline 27 & $\mathrm{PV}$ & MUT (71 \pm 2$)$ & BM & 78 & $46, X X$ & MUT $(69 \pm 2)$ & MUT (65 \pm 1.5$)$ & MUT $(68 \pm 2.3)$ & WT \\
\hline 28 & $\mathrm{PV}$ & MUT (59 \pm 1.5$)$ & BM & 90 & $46, X X$ & MUT $(58 \pm 1.75)$ & MUT (58 \pm 1.5$)$ & MUT (59 \pm 1.5$)$ & WT \\
\hline 29 & PMF & MUT (61 \pm 2$)$ & BM & 53 & ND & MUT $(60 \pm 1.15)$ & MUT $(60 \pm 1.5)$ & MUT $(59 \pm 1.75)$ & WT \\
\hline 30 & PV & MUT (49 \pm 3$)$ & BM & 72 & ND & WT & WT & WT & WT \\
\hline 31 & $\mathrm{PV}$ & MUT (58 \pm 3$)$ & BM & 22 & $\mathrm{ND}$ & MUT (58 \pm 2$)$ & MUT $(59 \pm 2)$ & MUT (59 \pm 0.95$)$ & WT \\
\hline 32 & PMF & MUT $(56 \pm 2)$ & $\mathrm{BM}$ & 66 & $46, X Y$ & MUT $(57 \pm 2.65)$ & MUT $(55 \pm 2)$ & MUT $(55 \pm 2.13)$ & WT \\
\hline 33 & PMF & WT & BM & 60 & $46, X Y$ & WT & WT & WT & WT \\
\hline 34 & ET & WT & $\mathrm{BM}$ & 77 & $\mathrm{t}(9 ; 12) ; \mathrm{TRI}(8) ; \mathrm{ISO}(17)$ & WT & WT & WT & WT \\
\hline
\end{tabular}

MPN, myeloproliferative neoplasia; BM, bone marrow; PB, peripheral blood; WT, wild type; MUT, V617F mutated; AB, allele burden; SD, standard deviation; MNCs, mononucleated cells; ND, not done; DEL(x), deletion; TRI(x), trikomayyises( 